\title{
INFLUÊNCIA DO DANO FOLIAR EM Senna alata (L.) ROXB EM SEU INVESTIMENTO REPRODUTIVO
}

\author{
Ana Clara S. Santos ${ }^{1}$, Anna Clara Chaves Ribeiro ${ }^{*}$, Guilherme Murilo Oliveira ${ }^{2}$, \\ ${ }^{1}$ Maíra Machado Giraldin e ${ }^{1}$ Saturnina Da Costa \\ ${ }^{1}$ Graduandos em Ecologia e Analise Ambiental pela Universidade Federal de Goiás \\ ²Engenheiro agrônomo da Universidade Federal de Goiás \\ *annaclararibeiro7@gmail.com
}

Recebido em: 08/09/2015 - Aprovado em: 14/11/2015 - Publicado em: 01/12/2015
DOI: http://dx.doi.org/10.18677/Enciclopedia_Biosfera_2015_265

\section{RESUMO}

Os herbívoros provocam diferentes tipos de danos nas folhas, caules, brotos, raízes, sementes e frutos. O dano foliar, portanto, é um dos fatores que influenciam o esforço reprodutivo da planta, pois está relacionado com a perda das folhas, que é o principal órgão fotossintetizante. Objetivou-se avaliar a influência do dano foliar induzido em Senna alata em seu esforço reprodutivo. Foram aplicados os gradientes de simulação de dano foliar, sendo tratamentos $0 \%, 25 \%, 50 \%$ e $75 \%$. O dano foliar simulado em Senna alata não influenciou os componentes do esforço reprodutivo, porém o número de folíolos influenciou a produção de frutos. A relação entre número de folíolos e a produção de frutos pode ser devido a produção e armazenamento prévio de energia, posteriormente utilizada para a produção de frutos.

PALAVRAS-CHAVE: esforço, frutos, semente, simulação, sucesso reprodutivo.

\section{DAMAGE INFLUENCE OF LEAF IN Senna alata (L.) ROXB IN YOUR INVESTMENT REPRODUCTIVE}

\begin{abstract}
Herbivores cause different types of damage on leaves, stems, buds, roots, seeds and fruits. The leaf damages therefore one of the factors that influence the reproductive effort of the plant because it is related to the loss of leaves, which is the main photosynthetic organ. Thus the present study aims to evaluate the influence of induced leaf damage on Senna alata in their reproductive effort. The foliar damage simulation gradients were applied, and treatments $0 \%, 25 \%, 50 \%$ and $75 \%$. Simulated leaf damage in $S$. alata did not influence the components of reproductive effort, but the number of leaflets affected the fruit production. The relationship between the number of leaflets and fruit production may be due to production and storage prior energy subsequently used for the production of fruit.
\end{abstract}

KEYWORDS: effort, fruit, seed, simulation, reproductive success. 


\section{INTRODUÇÃO}

O dano foliar é causado nas plantas por vários grupos de animais, tanto por vertebrados quanto por invertebrados, porém o principal grupo são os insetos, sendo que em florestas tropicais são responsáveis por danos entre $10-25 \%$ das partes vegetativas das plantas (STRAUSS e ZANGERL, 2002; GRIMALDI e ENGEL, 2005). Dentre os vários tipos de dano foliar, a folivoria é a mais fácil de registrar, pois ocorre na lâmina foliar, exceto quando toda a folha é removida e consumida pelo herbívoro (CORREA et al. 2008; COTARELLI e ALMEIDA, 2015). O sucesso reprodutivo das comunidades de plantas pode ser influenciado por mudanças abióticas causadas geralmente por fragmentação de hábitats e por predação insetívora de sementes (MELO-SILVA et al., 2014; FRANCESCHINELLI et al. 2015).

O dano foliar, portanto, é um dos fatores que mediam o sucesso reprodutivo, pois está relacionado com a perda das folhas, que é o principal órgão fotossintetizante da planta (ERDOGMUS, 2010). O padrão espacial dos danos também pode determinar o impacto da herbivoria sobre o desempenho da planta. É importante medir o padrão do dano, pois dano foliar em ramos com frutos tem um efeito negativo maior comparado com uma quantidade igual de danos em ramos distantes dos frutos (EDWARDS e WRATTEN, 1983).

O gênero Senna tem distribuição pantropical com mais de 350 espécies existentes, sendo 200 delas, ocorrendo na América e ao menos 60 no Brasil (BEGUN et al., 2014). Senna alata (L.) Roxb. é uma planta perene, arbustiva e é considerada uma espécie daninha, e apresenta crescimento vegetativo muito acelerado (RODRIGUES et al., 2009). Este crescimento acelerado provavelmente ocorre por consequências de produção abundante de sementes, pela alta taxa de reprodução (tanto sexuada como assexuada), florescimento precoce, dispersão facilitada, alta plasticidade, tolerância a diferentes condições climáticas, longo tempo de floração e frutificação (MARABESI, 2011; FARIA et al., 2013) dentre algumas adaptações e relações com o efeito do ambiente e do seu sucesso reprodutivo.

O dano foliar, quando distribuído nos ramos, pode reduzir a quantidade de frutos formados, pois as folhas proporcionam muitas vezes a maior parte dos recursos para o seu desenvolvimento (VIEIRA e LOMÔNACO, 2014). Apesar de alguns frutos serem fotossintetizantes durante seu crescimento, nem sempre a sua contribuição é suficiente para compensar a perda de fotossintato produzido quando há a perda de folhas. Para algumas culturas agronômicas, como o morangueiro, essas relações entre dano foliar e produção já são bem descritas (OTTO et al. 2012).

De acordo com o exposto acima, é definido a hipótese de que o dano foliar simulado poderia influenciar na produção de frutos e sementes, pois a perda de área foliar diminuiria a taxa fotossintética da planta, e assim ofereça menos recurso energético para o desenvolvimento dos frutos e sementes. Assim, objetivou-se avaliar a influência do dano foliar em $S$. alata na taxa de produção de frutos e sementes, simulando-o em três gradientes.

\section{MATERIAIS E MÉTODOS}

\section{Área de estudo}

Os doze espécimes de $S$. alata, de aproximadamente dois anos de idade, se

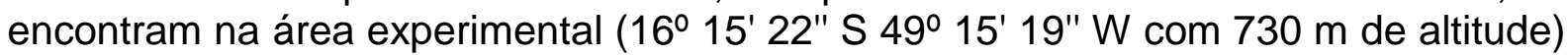


em solo com textura média e classificado como Latossolo Vermelho Perférrico distrófico (SANTOS et al., 2006), próximo a área de fragmento de floresta semidecídua secundária.

\section{Experimento}

Foram analisados doze espécimes de Senna alata antes e durante o período de floração e frutificação (abril a junho de 2015). Cada indivíduo teve seus galhos secundários enumerados. Houve o sorteio de quatro ramos de cada indivíduo para que fossem aplicados os gradientes de simulação de dano foliar. Os tratamentos consistiram em 0\% (controle - sem dano SD); 25\%, 50\% e 75\% (Figura 01) (Adaptado de VIEIRA e. LOMÔNACO, 2014).

A folha de $S$. alata é composta desta forma os gradientes de desfolhamento foram proporcionais ao número total de folíolos de cada folha, sendo no gradiente de dano de $25 \%$ em um galho com 28 folíolos foram retirado sete folíolos, no gradiente de dano de $50 \%$ retirado 14 folíolos e $75 \%$ retirado 21 folíolos.
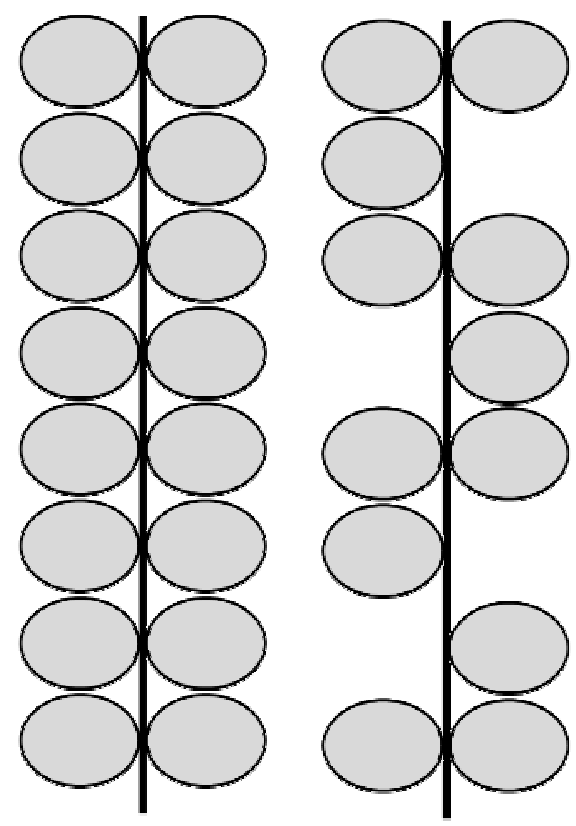

$25 \%$

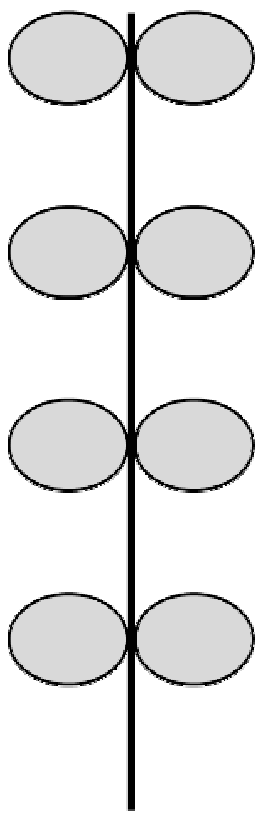

$50 \%$

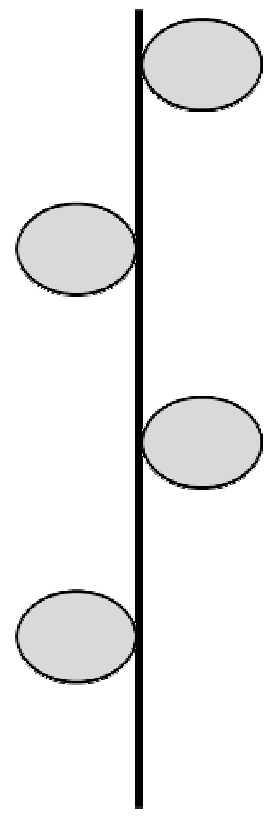

$75 \%$

(Controle)

Figura 01. Representação de dano foliar simulando herbivoria nos folíolos de Senna alata, onde: a) SD (sem dano), tratamento controle; b) $25 \%$ de dano foliar; c) $50 \%$ de dano foliar e d) $75 \%$ de dano foliar.

Os dados coletados para quantificar o investimento reprodutivo (variáveis respostas) foram: o número de botões florais por ramo, o número flores por ramo, o número de frutos por ramo, $\mathrm{n}^{\circ}$ de sementes, taxa de produção de sementes (número de sementes pelo número de flores) e taxa de produção de flor (número de flores por número de botões florais). O número de frutos e de sementes foi contabilizado após um período de três semanas do momento de desfolhamento.

\section{Analise estatística}


Para avaliação estatística foi realizado correlação de Spearman, comparando os tratamentos (controle, $25 \%, 50 \%$ e $75 \%$ ), individuo (1 a 12) e número de folíolo com as variáveis respostas. Posteriormente da verificação da ausência da correlação entre as variáveis foi realizado regressão múltipla com nível de significância estatística de $95 \%$.

Os valores de significância e os coeficientes de determinação $\left(R^{2}\right)$ foram observados para buscar os melhores modelos. Para a variável que apresentou significância foi criado representação gráfica da regressão linear entre as variáveis.

\section{RESULTADOS E DISCUSSÃO}

O número de botões florais por ramo, o número flores por ramo, o número de frutos por ramo, $\mathrm{n}^{\circ}$ de sementes, taxa de produção de sementes (número de sementes pelo número de flores) e taxa de produção de flor (número de flores por número de botões florais, não apresentaram correlação $(p>0,05)$.

$O$ dano foliar simulado em $S$. alata não influenciou os componentes do esforço reprodutivo da espécie $(p<0,05)$ como número de frutos e sementes. Porém, o número de folíolos de cada ramo da planta influenciou a produção de frutos por ramo $\left(p<0,001 ; R^{2}=0,44\right)$ (Tabela 01; Figura 02). Assim, a cada unidade de folíolo acrescentada no ramo, houve o aumento na produção de frutos em $16 \%$.

Tabela 01. Regressão múltipla realizada entre número de indivíduos, dano foliar e $\mathrm{n}^{\circ}$ de folíolos entre botões florais por ramo, flores por ramo, frutos por ramo, $\mathrm{n}^{\circ}$ de sementes, taxa de produção de sementes e taxa de produção de flor $(F(18,110)=0,435 ; p=0,01)$.

\begin{tabular}{|c|c|c|c|c|c|c|}
\hline $\begin{array}{c}\text { Variáveis } \\
\text { independentes }\end{array}$ & $\begin{array}{c}\text { Variáveis } \\
\text { dependentes }\end{array}$ & $\begin{array}{l}\text { Coef. } \\
\text { de } \\
\text { regressão }\end{array}$ & $\begin{array}{l}\text { Desvio } \\
\text { do erro }\end{array}$ & $\mathbf{t}$ & $\mathbf{p}$ & $\begin{array}{c}\text { Coef. } \\
\text { de } \\
\text { determinação } \\
\left(\mathbf{R}^{2}\right)\end{array}$ \\
\hline \multirow[t]{7}{*}{ Indivíduos } & & 11,54 & 7,09 & 1,627 & 0,111 & \\
\hline & Botões por ramo & 0,019 & 0,02 & 0,944 & 0,350 & 0,029 \\
\hline & Flores por ramo & $-0,064$ & 0,05 & $-1,216$ & 0,230 & 0,072 \\
\hline & Frutos por ramo & $-0,068$ & 0,07 & $-0,959$ & 0,343 & 0,061 \\
\hline & Sementes & 0,018 & 0,02 & 0,890 & 0,378 & 0,001 \\
\hline & Taxa de semente & $-0,182$ & 0,20 & $-0,877$ & 0,385 & 0,023 \\
\hline & Taxa de flor & 2,488 & 3,80 & 0,654 & 0,516 & 0,003 \\
\hline \multirow[t]{7}{*}{ Dano foliar } & & 62,943 & 59,37 & 1,060 & 0,295 & \\
\hline & Botões por ramo & $-0,278$ & 0,17 & $-1,620$ & 0,112 & 0,007 \\
\hline & Flores por ramo & 0,554 & 0,44 & 1,244 & 0,220 & 0,0003 \\
\hline & Frutos por ramo & 0,235 & 0,59 & 0,394 & 0,695 & 0,0002 \\
\hline & Sementes & $-0,022$ & 0,17 & $-0,128$ & 0,898 & 0,003 \\
\hline & Taxa de semente & $-0,329$ & 1,74 & $-0,188$ & 0,851 & 0,001 \\
\hline & Taxa de flor & $-33,361$ & 31,82 & $-1,048$ & 0,300 & 0,006 \\
\hline \multirow[t]{7}{*}{$\mathrm{N}^{\circ}$ de folíolos } & & 22,908 & 3,70 & 6,180 & 0,000 & \\
\hline & Botões por ramo & $-0,005$ & 0,01 & $-0,493$ & 0,624 & 0,064 \\
\hline & Flores por ramo & 0,014 & 0,02 & 0,523 & 0,603 & 0,143 \\
\hline & Frutos por ramo & 0,162 & 0,03 & 4,369 & 0,000 & 0,440 \\
\hline & Sementes & $-0,017$ & 0,01 & $-1,562$ & 0,125 & 0,015 \\
\hline & Taxa de semente & 0,039 & 0,10 & 0,366 & 0,716 & 0,018 \\
\hline & Taxa de flor & 0,958 & 1,98 & 0,482 & 0,632 & 0,057 \\
\hline
\end{tabular}




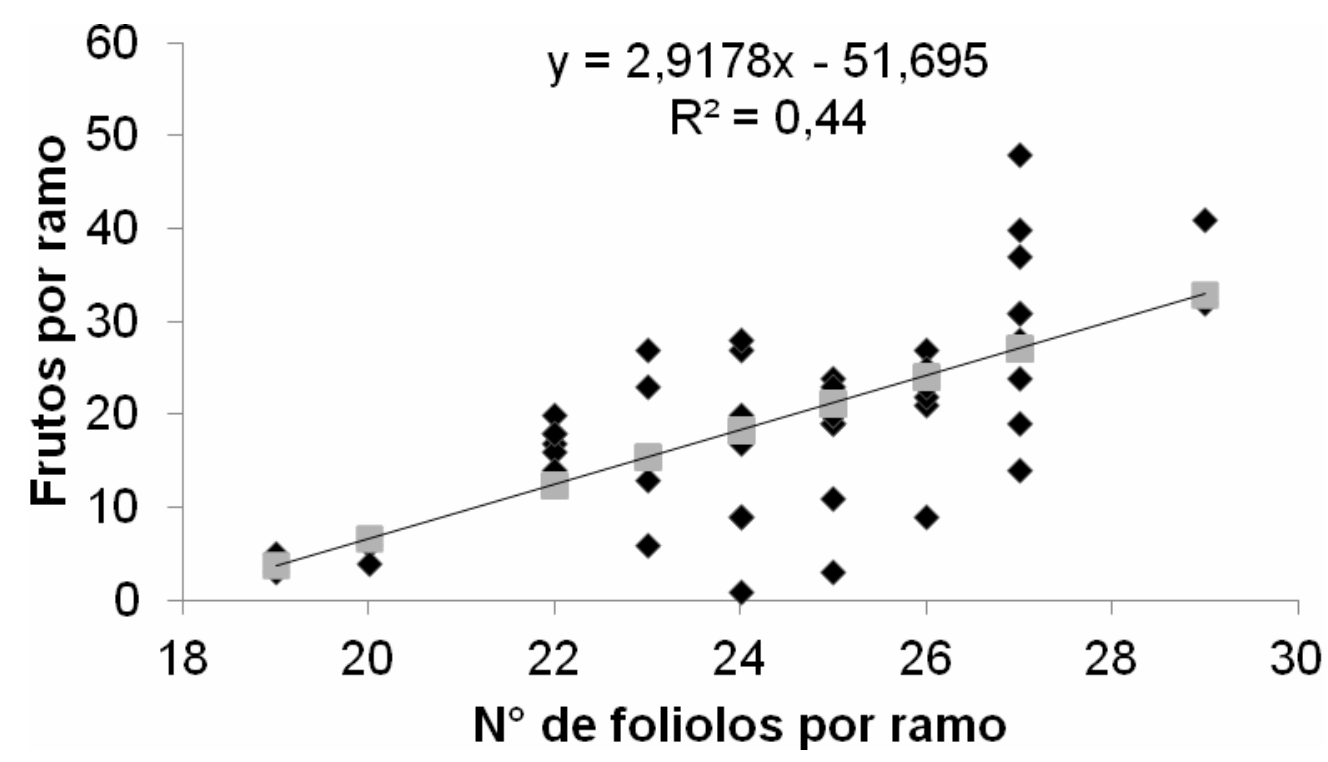

Figura 02. Correlação de Spearman para número de folíolos (eixo $x$ ) e número de frutos finais (eixo y) $\left(y=2,9178 x-51,695 ; R^{2}=0,44 ; p<0,001\right)$.

A hipótese que o dano foliar simulado em ramos de Senna alata influencia no seu investimento reprodutivo, bem como na produção de frutos e sementes não foi corroborada, pois não houve diferença entre os danos aplicados para a produção de sementes. A falta de efeito pode ser ocasionada por dois fatores: (i) a fisiologia da planta não ser setorial e sim por todo o indivíduo, (ii) compensação fotossintética dos folíolos restantes ou outra forma de energia vinda de outros metâmeros da planta (VALLADARES et al 2007). RONQUIM et al. (2013) evidenciam que Vochysia tucanorum Mart. apresenta adaptações de acordo com as alterações ambientais, podendo assim, desenvolver-se nas novas condições estabelecidas.

O dano foliar pode ser um estresse para as plantas, cuja intensidade é dependente da dimensão da desfolha. Quando ocorre a desfolha há uma menor intercepção de luz e consequentemente de fotossíntese e uma queda na quantidade de compostos orgânicos de reserva e crescimento das raízes (GOMIDE et. al., 2002). Porém o transporte de energia e nutrientes na planta, não é somente da base do caule/galhos da planta, mas também das raízes em favor dos novos tecidos e da produção de frutos. A relação positiva com o número de folíolos no galho e a produção de frutos pode ser devido a produção e armazenamento prévio de energia, posteriormente utilizada para a produção de frutos e o esforço reprodutivo.

A compensação dos danos por meio ocasiona uma produção de maiores estruturas, podendo assim atingir uma maior biomassa total do que os que não foram danificados (VALLADARES et al 2007). As respostas compensatórias são flexíveis e podem ser influenciadas por condições bióticas e abióticas (BRUNA e RIBEIRO, 2015).

As experiências feitas por TUOMI et al. (1988) mostraram que a maior parte do carbono necessário para o desenvolvimento dos frutos em Betula pubescens Ehrh., vem de reservas armazenadas em anos anteriores. Em contraste, HAUKIOJA et al., (1990), mostrou que o dano foliar em B. pubescens afetou o crescimento apenas um ano após. Isto sugere que a separação temporal do tempo de acumulação de 
recursos e uso do mesmo não confere as restrições de movimento dos recursos limitados entre os ramos.

De acordo com SHEA e WATSON (1989) foi provado que o movimento de carbono é restrito dentro do arranjo foliar. Porém, ao contrário de suas previsões, a frutificação foi igual para os tratamentos, distribuindo os danos entre os ramos. Todos os tratamentos reduziram a produção em comparação às plantas controle não danificadas. Este resultado mostra que a sequência de dano aumenta o movimento de recurso entre ramos danificados e não danificados.

É importante levar em consideração que a interação entre fenologia de floração entre outras relações nutricionais em meio às estruturas em desenvolvimento, influencia fortemente as propriedades no resultado do número frutos e sementes (BAWA e WEB, 1984). COTARELLI E ALMEIRA (2015) evidenciando a florivoria em $S$. macranthera (Colladon) Irwin \& Barneby, verificaram relação negativa com seu sucesso reprodutivo, uma vez que abelhas não visitam flores com os herbivoros.

Para jatobá da mata (Hymenaea courbaril L.), as plantas jovens submetidas ao dano foliar apresentaram folhas com limbos mais espessos, característica de defesa induzida para reduzir a palatabilidade foliar. A modificação foliar induzida está associada a um custo energético, refletido na redução da taxa de crescimento da planta e na diminuição das dimensões da nervura foliar central e provavelmente no esforço reprodutivo (VIEIRA e LOMÔNACO, 2014). Dentre algumas culturas de interesse agronômico, como o morangueiro (Fragaria $\mathrm{x}$ ananassa Duch.), há grande preocupação com o dano foliar, provocado por herbívoros ou outras causas, como a ocorrência de granizo. Nesta situação, os autores verificam relação inverna entre o aumento do dano foliar e a redução do número e tamanho de frutos (OTTO et al. 2012).

\section{CONCLUSÃO}

O esforço reprodutivo de Senna alata não é influenciado pelo dano foliar simulado, mas sim pelo número de folíolos, podendo indicar resposta da espécie contra herbívoros.

\section{REFERÊNCIAS BIBLIOGRÁFICAS}

BAWA, K. S.; WEBB, C. J. Flower, Fruit and Seed Abortion in Tropical Forest Trees: Implications for the Evolution of Paternal and Maternal Reproductive Patterns. American Journal of Botany, v. 71, n. 5, p. 736-751, 1984.

BEGUN, A.; RAHMAN, O; BEGUN, M. Stomatal and trichome diversity in Senna Mill. From Bangladesh. Bangladesh Journal of Plant Taxonomy, v. 21, n. 1, p. 43-51, 2014.

BRUNA, E. M., RIBEIRO, M. B. N. The compensatory responses of an understory herb to experimental damage are habitat-dependent. American Journal of Botany, v. 92 , n. 12 , p. 2101-2106, 2005.

COTARELLI, C. M.; ALMEIDA, N. M. Florivoria em Senna macranthera var. 
pudibunda (Benth.) H.S.Irwin \& Barneby (Caesalpinioideae-Fabaceae). Natureza on line, v. 13, n. 1, p. 45-49, 2015.

CORREA, P. G.; PIMENTEL, R. M. M.; CORTEZ, J. S. A.; XAVIER, H. S. Herbivoria e anatomia foliar em plantas tropicais brasileiras. Ciência e Cultura, v. 60, n. 3, p. 54-57, 2008.

DANTAS, M. M.; SILVA, M. J. O gênero Senna Mill. (Leguminosae, Caesalpinioideae, Cassieae) no Parque Estadual da Serra Dourada, GO, Brasil. Hoehnea, v. 40, n. 1, p. 99-113, 2013.

EDWARDS, P. J.; WRATTEN, S. D. Wound induced defenses in plants and their consequences for patterns of insect grazing. Oecologia, v. 59, s/n, p. 88-93, 1983.

FARIA, J. C. T.; CALDEIRA, M. V. W.; DELARMELINA, W. M.; LACERDA, L. C.; OLIVEIRA-GONÇALVES, E. Substratos à base de lodo de esgoto na produção de mudas de Senna alata. Comunicata Scientiae, v. 4, n. 4, p. 342-351, 2013.

FRANCESCHINELLI, E. V.; CARMO, R. M.; SILVA-NETO, C. M.; GONÇALVES, B. B.; BERGAMINI, L. The reproductive success of Cabralea canjerana (Vell.) Mart. (Meliaceae) in Atlantic Forest fragments in Brazil. Revista de Biologia Tropical, v. 63, n. 1, p. 515-524, 2015.

GOMIDE, M. A. C.: GOMIDE, A. J.; HUAMAN, M. A. C.; PACIULLO, C. S. D. Fotossíntese, Reservas Orgânicas e Rebrota do Capim-Mombaça (Panicum maximum Jacq.) sob Diferentes Intensidades de Desfolha do Perfilho Principal. Revista Brasileira de Zootecnia, v.31, n.6, p. 2165-2175, 2002

GRIMALDI, D.; ENGEL, M. S. Evolution of the insects. United Kingdom: Cambridge University Press, 2005. 733 p.

HAUKIOJA, E.; RUOHOMIIKI, K.; SENN, J.; SUOMELA, J.; WALLS, M. Consequences of herbivory in the mountain birch (Betula pubescens ssp. tortuosa): importance of the functional organization of the tree. Oecologia, v. 82, n. 2, p. 238247, 1990.

LOWMAN, M. D. Leaf growth dynamics and herbivory in five species of Australian rain-forest canopy trees. Journal of Ecology, v. 80, n. 3, p. 433-447, 1992.

MELO-SILVA, C.; PERES, M. P.; MESQUITA-NETO, J. N.; GONÇALVES, B. B.; LEAL, I. A. B. Biologia reprodutiva de Leucaena leucocephala (Lam.) R. de Wit (Fabaceae: Mimosoideae): sucesso de uma espécie invasora. Neotropical Biology and Conservation, v. 9, n. 2, p. 91-97, 2014.

OTTO, F.; OHSE, R. S.; CORSO, F. Estimativa de danos de granizo no limbo foliar do morangueiro sob proteção do agrotêxtil. Idesia, v. 30, n. 2, p. 39-43, 2012.

RONQUIM, C. C.; ASSIS-PRADO, C. H. B.; SOUZA, J. P. Estabelecimento e crescimento de plantas jovens de Vochysia tucanorum Mart.(Vochysiaceae) em área 
aberta e sombreada sob dossel florestal. Scientia Forestalis, v. 41 , n. 97 p. 121130, 2013.

SANTOS, H. D.; JACOMINE, P. K. T.; ANJOS, L. D.; OLIVEIRA, V. D.; OLIVEIRA, J. D.; COELHO, M. R.; CUNHA, T. D. Sistema brasileiro de classificação de solos. EMBRAPA - Empresa Brasileira De Pesquisa Agropecuária. Rio de Janeiro, 2006. $412 p$.

SHEA, M. M.; WATSON, M. A. Patterns of leaf and flower removal: their effect on fruit growth in Chamaenerion angustifolium (fireweed). American Journal of Botany, v. 76, n. 6, p. 884-890, 1989

STRAUSS, S. Y.; AGRAWAL, A. A. Ecology and evolution of plant tolerance herbivory. Trends in Ecology and Evolution, v. 53, n. 4, p. 1093-1104, 1999.

TUOMI, J.; NISULA, S.; VUORISALO, T.; NIEMELI, P.; JORMALAINEN, V. Reproductive effort of short shoots in silver birch (Betula pendula Roth). Experientia, v. 44, n. 6 , p. $540-541,1988$.

VALLADARES, F.; GIANOLI, E.; GÓMEZ, J. M. Ecological limits to plant phenotypic plasticity. New Phytologist, v. 176, n.4, p. 749-763, 2007.

VIEIRA, A. O.; LOMÔNACO, C. Responses of young plants of Hymenaea courbaril L. (Fabaceae) to damages caused by herbivory simulation. Bioscience Journal, v. 30, n. 4, p. 1222-1230, 2014.

WATSON, M. A.; CASPER, B. B. Morphogenetic constraints on patterns of carbon distribution in plants. Annual Review of Ecology and Systematics, v. 15, n. s/n, p. 233-258, 1984. 